\title{
Polibio e gli imperi (Filippo V, Cartagine e altri paradeigmata) ${ }^{1}$
}

\author{
John ThORNTON \\ "Sapienza" Università di Roma \\ Dipartimento di Scienze dell'Antichità \\ john.thornton@uniroma1.it
}

Nel titolo di questo incontro, alle relazioni culturali sono opportunamente affiancate quelle diplomatiche. Nel mondo mediterraneo, nell' antichità, lo sguardo degli altri non è solo lo sguardo dell'osservatore distaccato; spesso è lo sguardo interessato di chi persegue finalità politiche.

L'immagine quintilianea della storiografia assimilabile all'epidittica, e composta non ad actum rei pugnamque praesentem sed ad memoriam posteritatis et ingenii famam (Institutio oratoria $\mathrm{X} 1,31$ ), non corrisponde all'attività del politico acheo cui è dedicata questa seduta; da Polibio, non ci si potrebbe attendere una storiografia above the battle. Non era stata tale, d'altra parte, e non si sarebbe potuta assimilare all'epidittica né la storiografia di Democare, il combattivo nipote di Demostene che aveva accusato con parrhesia Antipatro e Demetrio del Falero ${ }^{2}$, né quella di Filarco, che Plutarco poté assimilare all'oratoria giudiziaria per il carattere della narrazione del confronto fra Arato

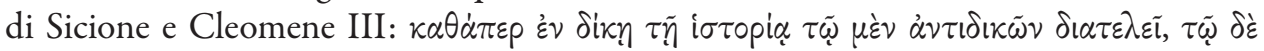

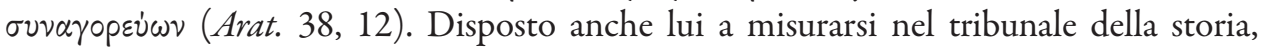
davanti alla giuria dei lettori, Polibio assunse la difesa di Arato, sforzandosi di dimostrare

1 Desidero ringraziare l'amica Maria Teresa Schettino e Antonio Gonzales per lo stimolante invito a partecipare alla rencontre, e per la calorosa ospitalità a Mulhouse e Strasburgo. La relazione, pubblicata qui con qualche lieve ampliamento e un corredo di note assai contenuto, riprende e sviluppa quella già presentata nell'ottobre 2008, a Firenze, al Convegno internazionale di studio Scrittori antichi e imperi moderni, organizzato dal professor Paolo Desideri, e rientra in una più ampia indagine sulla dimensione politica dell'opera di Polibio.

2 Cicerone Brutus 286; Polibio XII 13, 7-9. 
la falsità della versione di Filarco; e dispiegò un dominio delle tecniche retoriche non inferiore rispetto al suo rivale ${ }^{3}$.

L'educazione retorica pervade infatti la cultura di Polibio, che è capace di impiegare con naturalezza le lezioni apprese alla scuola dei retori non solo nella polemica con i suoi predecessori ${ }^{4}$, ma anche nelle trattative con le autorità romane e di fronte alle assemblee achee. Al tempo della terza guerra di Macedonia, persuase i suoi connazionali a votare per la restituzione degli onori al re di Pergamo Eumene II $^{5}$; e più tardi, al termine della guerra acaica, difese con successo gli onori di Filopemene davanti alla commissione senatoria che collaborò con Lucio Mummio ${ }^{6}$. Prestare attenzione alla dimensione retorica della formazione di Polibio (e dei suoi lettori contemporanei) potrà consentire di intendere, spero, il senso più profondo anche delle considerazioni di Polibio sugli imperi. In questa relazione, ci si interrogherà dunque sulla dimensione performativa delle Storie: si tenterà cioè di indagare gli obiettivi perseguiti dall'autore attraverso il sapiente ricorso alle armi della retorica ${ }^{7}$.

L'opera che meglio può contribuire a illuminare le strategie retoriche adottate nelle Storie, perché più sembra potersi avvicinare al 'manuale' su cui studiò Polibio, è senza dubbio la Rhetorica ad Alexandrum. Nella sua trattazione dei paradeigmata, definiti

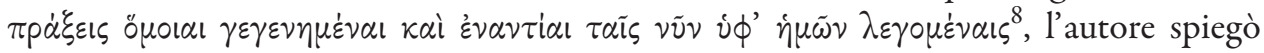
la natura di quelli $\dot{\varepsilon} \kappa \tau \tilde{\omega} \nu \dot{\varepsilon} \nu \alpha \nu \tau i \omega \nu$, prevedibilmente, con un esempio: $\lambda \dot{\varepsilon} \gamma \omega \delta^{\prime}$ oíov $\dot{\varepsilon} \dot{\alpha} \nu$

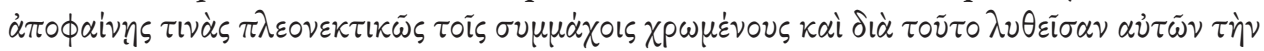

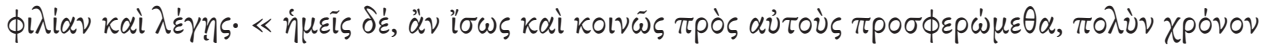

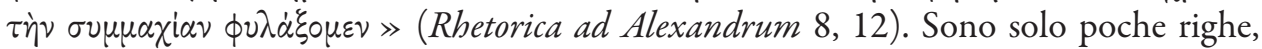
sufficienti però ad attestare come già a un livello cronologico anteriore a quello di Polibio fosse comunemente ammesso che la menzione di casi - esempi, appunto, paradeigmata in cui l'arroganza nei confronti degli alleati aveva portato alla dissoluzione dell' amicizia

3 J. Thornton, Tragedia e retorica nella polemica sulla presa di Mantinea (Polibio II, 56-58), in M. Mari e J. Thornton (a cura di), Parole in movimento. Linguaggio politico e lessico storiografico nel mondo ellenistico. Atti del Convegno Internazionale, Roma, 21-23 febbraio 2011, Studi Ellenistici, XXVII, a cura di B. Virgilio, Pisa-Roma 2013, p. 353-374.

4 J. Thornton, Oratory in Polybius' Histories, in K. Tempest and Chr. Kremmydas (ed. by), Hellenistic Oratory: Continuity and Change, Oxford 2013, p. 21-42; ma naturalmente vd. già, con prospettiva assai più ampia, M. Isnardi,

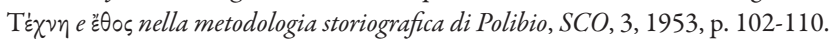

5 Polibio XXVIII 7, con Thornton, Oratory in Polybius' Histories, op. cit., p. 31-33.

6 Polibio XXXIX 3.

7 Cf. già J. Thornton, Polybius in Context. The Political Dimension of the Histories, in B. Gibson, Th. Harrison (ed. by), Polybius and his World. Essays in Memory of F.W. Walbank, Oxford, 2013, p. 213-229.

8 [Aristotele], Rhetorica ad Alexandrum 8, 1 (1429a 21-22). 
poteva essere finalizzata ad esortare la potenza cui ci si rivolgeva a trattare i propri alleati su un piano di parità. La formulazione del trattato di retorica esprime la cultura condivisa da Polibio e dal suo pubblico, accomunati dai problemi contemporanei e, appunto, dall'educazione retorica. Non c'è quasi bisogno di rilevare che l'alleanza di cui si parla è un'alleanza di tipo egemonico: solo in una relazione caratterizzata da un notevole squilibrio nei rapporti di forza può sorgere la tentazione di trattare i propri alleati con arroganza. Per mantenerne l'amicizia, per assicurare cioè la durata della propria egemonia,

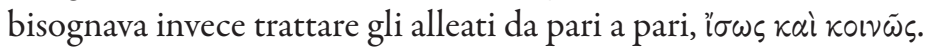

Nelle Storie, a questo proposito, s'incontrano non solo paradeigmata $\dot{\varepsilon} \kappa \tilde{\omega} \nu$ $\dot{\varepsilon} \nu \alpha \nu \tau i \omega \nu$, ma anche esempi $\dot{\varepsilon} \kappa \tau \tilde{\omega} \nu$ ó $\mu o i \omega \nu$ : Polibio non si limita a registrare i casi di quanti avevano perso l'egemonia per aver trattato gli alleati con superbia, ma celebra anche il modello di quanti invece con la magnanimità avevano saputo procurarsi l'eunoia sincera dei popoli soggetti. Simili paradeigmata ricorrono nelle Storie in modo quasi ossessivo; è forte l'impressione che Polibio intendesse impartire la stessa lezione delineata nel passo della Rhetorica ad Alexandrum.

D'altra parte, riflettendo sul posto dei discorsi nella storiografia, a partire dalla critica a Timeo, in due brani del XII libro Polibio aveva affermato la necessità che l'uso della storia divenisse utile, fruttuoso, है $\gamma$ kap $\pi \circ \varsigma$. E ciò sarebbe potuto accadere riportando alla propria epoca le circostanze simili riferite dallo storico, che avrebbe dovuto mostrare anche « la ragione per cui ciò che fu fatto o detto fallì o riuscì » ${ }^{9}$. La funzione della storia come deposito di esempi commentati da metapherein epi toùs oikeious kairoùs, o epi tá paraplesia, per « riuscire sempre a conseguire i nostri obiettivi ${ }^{10}$, ricorda il compito dell'oratore, che si sforza di indirizzare le decisioni politiche attraverso gli opportuni paradeigmata.

\section{$-1-$}

In IX 23, 5-9 Polibio considerava la possibilità che in certi casi i comportamenti di Annibale fossero stati influenzati dai suoi consiglieri; quindi, passava a riflessioni più generali.

Lo stesso accade di solito anche per effetto dei suggerimenti degli amici, non solo ai capi, ai sovrani e ai re, ma anche alle città. Tra gli Ateniesi, per esempio, si potrebbero trovare

\footnotetext{
9 Polibio XII 25b, 1-3.

10 Polibio XII 25i, 8. Sulla funzione didattica della storiografia in Polibio ha opportunamente insistito, di recente, M.-R. Guelfucci, Polybe et les mises en scène de la Tychè, DHA supplément, 4.2, Besançon 2010, p. 439-468.
} 
poche durezze e molti atti buoni e degni di rispetto quando Aristide e Pericle erano alla guida dello stato, il contrario con Cleone e Carete; quando gli Spartani avevano l'egemonia sulla Grecia, tutte le iniziative del re Cleombroto rispettavano i princìi dell'alleanza, al contrario di quelle di Agesilao; così, anche i costumi delle città mutano in accordo con le differenze tra i governanti. Il re Filippo, quando suoi collaboratori erano Taurione o Demetrio, era il più empio degli uomini, il più mite, invece, quando cooperavano con lui Arato o Crisogono ${ }^{11}$.

Già la contrapposizione di Aristide e Pericle a Cleone e Carete potrebbe forse riguardare in primo luogo l'atteggiamento verso gli alleati, e in particolare la determinazione e l'esazione dei tributi ${ }^{12}$. In ogni caso, è innegabile che qui Polibio vada progressivamente focalizzando l'attenzione sul tema dell'egemonia, centrale per lui e i suoi contemporanei, e che individui due possibili linee di condotta per una potenza imperiale: ad una hairesis symmachikè, rispettosa dei diritti degli alleati, se ne contrappone una caratterizzata dalla pikría, la durezza nei loro confronti.

Per il lettore delle Storie, a fornire immediatamente la chiave per intendere il senso di questa analisi doveva essere il riferimento all'opposta influenza esercitata sul giovane Filippo V dai suoi consiglieri. La giustapposizione di Arato e Demetrio rimanda infatti direttamente a un celebre brano del VII libro. In VII 12, Polibio narrava della tentazione del re di occupare militarmente l'Itome, nel 215 o 214 a.C., dopo aver svolto un ruolo per noi non del tutto chiaro, ma certo condannato da Arato e da Polibio, nel sanguinoso conflitto civile a Messene. Compiuto un sacrificio a Zeus sull'Itome, Filippo $\mathrm{V}$ aveva chiesto ai suoi consiglieri se ritenessero che avrebbe dovuto occupare la posizione. Demetrio gli raccomandò di comportarsi da basileús pragmatikòs, cogliendo l'opportunità di sottomettere il Peloponneso: solo impugnandone saldamente le corna - l'Acrocorinto e l'Itome - avrebbe avuto in suo potere il toro. La risposta di Arato fu di segno diametralmente opposto:

« Se puoi impadronirti di questo posto senza tradire i patti con i Messenii, ti consiglio di farlo; ma se, dopo averlo occupato con una guarnigione, sei destinato a perdere tutte le acropoli e la guarnigione dalla quale erano sorvegliati gli alleati che hai ricevuto in eredità da Antigono » - si riferiva alla lealtà (pistis) - « considera se ora non sia meglio portar

11 Le traduzioni polibiane sono tratte, qui e altrove, dall' edizione a cura di D. Musti, Polibio, Storie, I-VIII. Traduzione di M. Mari (libri I-XVIII; XXVIII-XXXIII e frammenti), F. Canali de Rossi (libri XIX-XXVII), A. L. Santarelli (libri XXXIV-XXXIX). Note di J. Thornton, Milano, I-VIII, 2001-2006.

12 In questa sede, non è opportuno indugiare su questo aspetto; cf. però G. Zecchini, Polibio e la storia non contemporanea, in P. Desideri, S. Roda e A.M. Biraschi con la collaborazione di A. Pellizzari (a cura di), Costruzione e uso del passato storico nella cultura antica. Atti del convegno internazionale di studi Firenze 18-20 settembre 2003, Alessandria, 2007, p. 213-223, in particolare 215. 
fuori gli uomini e lasciare qui la lealtà, e con questa sorvegliare i Messenii, come fai con gli altri alleati ».

L'alternativa presentata a Filippo dai suoi consiglieri sulla rocca di Messene riguarda l'atteggiamento più conveniente da assumere nei confronti degli alleati minori: Demetrio riteneva che un basileus pragmatikòs dovesse imporsi con la forza; secondo Arato, l'unica via praticabile era procurarsi il favore degli alleati grazie allo scrupoloso rispetto della lealtà nei loro confronti.

Questa stessa alternativa si era presentata più volte a Filippo V, nei suoi primi anni di regno; e Polibio non si lascia sfuggire l'occasione di commentare le scelte del re, e di illustrare le opzioni che gli si aprivano attraverso il ricorso a paradeigmata derivati dalla storia della dinastia macedone. $\mathrm{Nel} 220$, alla vigilia della guerra sociale contro gli Etoli, a Sparta scoppiarono dei disordini in cui rimasero uccisi i leaders filomacedoni; i responsabili tuttavia si affrettarono ad inviare un'ambasceria a Filippo V, garantendogli di voler rispettare scrupolosamente i termini del trattato. Fra i philoi di Filippo, alcuni, consapevoli dell'insincerità e della malizia degli ambasciatori, gli suggerirono di fare degli Spartani un paradeigma, « trattandoli nello stesso modo in cui Alessandro aveva trattato i Tebani, subito dopo aver preso il potere $\gg^{13}$. Il re scelse la linea opposta, che Polibio si affretta ad attribuire ad Arato ${ }^{14}$, e lasciò correre l'accaduto, richiamandosi a un ben diverso precedente, quello della magnanimità di Antigono Dosone dopo la vittoria di Sellasia: « sarebbe stato assurdo che mentre suo padre, quando da nemici li aveva sconfitti, non aveva fatto loro nulla di terribile, egli invece, per una colpa così piccola, prendesse riguardo a loro una decisione irreparabile $\gg$ (IV 24, 7). In questo modo, osserva Polibio, il re diede agli alleati una bella dimostrazione (kalón deigma) della sua proairesis. La decisione di Filippo V si presenta dunque come una scelta fra opposti paradeigmata: la linea del terrore e della durezza, esemplificata dalla distruzione di Tebe da parte di Alessandro nelle parole dei consiglieri di Filippo $V$ come poi in due celebri capitoli diodore ${ }^{15}$, e quella della mitezza, ricondotta ancora una volta ad Arato, e ispirata alla generosità di Antigono Dosone nei confronti degli Spartani vinti.

I modellidicomportamento opposti fra iquali si muove Filippo Vvengono illustrati ancor più diffusamente nel commento alla sua audace incursione a Termo, il santuario federale degli Etoli, nel 218. Penetrati nel cuore dell'Etolia, i Macedoni non si limitarono

\footnotetext{
13 Polibio IV 23, 8.

14 Polibio IV 24, 1-3 (« ... si potrebbe ascrivere ad Arato, con le maggiori probabilità, il parere espresso allora dal re »).

15 Diodoro Siculo XXXII 2 e 4, un brano molto discusso, anche riguardo al problema del rapporto con Polibio, sul quale però qui non ci si può soffermare.
} 
a saccheggiare l'area sacra, ricavandone un ricco bottino, ma distrussero sistematicamente gli edifici e i doni votivi; solo le statue degli dèi furono risparmiate. Il re e i suoi philoi, soddisfatti, ritenevano di aver vendicato appropriatamente le precedenti, empie scorrerie degli Etoli a Dion e Dodona. Polibio invece non approva il comportamento di Filippo, e si sofferma a spiegare ai lettori il senso della sua condanna ricorrendo a paradeigmata tratti dalla stessa casa reale macedone ( $\mathrm{V}$ 9, 8-10, 8): un procedimento che ritorna ad esempio nel discorso dell'etolo Alessandro Isio, che nei colloqui del golfo Maliaco, nel novembre del 198, rimproverò a Filippo di essersi allontanato dalle nobili tradizioni di Alessandro e dei diadochi ${ }^{16}$.

Il primo ad essere evocato è lo stesso esempio che già aveva ispirato la rinuncia a intervenire a Sparta, nel 220: sconfitto Cleomene III, Antigono non punì gli Spartani - sostiene Polibio -, ma restituì loro la libertà e il sistema politico tradizionale; in cambio, ottenne i titoli di evergete e salvatore, e onore e fama immortali presso tutti i Greci. Quindi, risalendo nel tempo, Filippo II: dopo la vittoria di Cheronea, Filippo rilasciò i prigionieri senza riscatto e rese gli onori funebri ai caduti ateniesi. Così, con l'epieikeia e la philanthropia riportò risultati maggiori di quelli conseguiti con le armi, trasformando gli Ateniesi da nemici in collaboratori pronti a tutto. In poche righe di testo, Polibio attribuisce a Filippo II le virtù dell'epieikeia, della philanthropia, dell'eugnomosyne, della metriotes, della praotes, della kalokagathia e della megalopsychia; la scelta di applicarle agli Ateniesi vinti viene considerata frutto di anchinoia, apprezzata cioè non solo sul piano morale, ma anche su quello dell'efficacia pratica ${ }^{17}$. Con sorprendente virtuosismo retorico, fra i paradeigmata utili a condannare il comportamento di Filippo V, e la giustificazione ricercatane nella vendetta, Polibio arruola poi anche Alessandro, che a Tebe non aveva spinto la sua ira fino all'empietà, e persino nella spedizione in Asia, pur intendendo vendicare l'asebeia dei Persiani contro i Greci, « risparmiò tutto quello che era stato consacrato agli dei, sebbene le colpe dei Persiani in Grecia fossero state commesse soprattutto in questo ambito ».

Sul metro di questi esempi, Polibio misura le scelte di Filippo V (V 11, 7-12, 3). Per conseguire la stessa fama dei suoi predecessori argeadi, di cui rivendicava l'eredità, Filippo ne avrebbe dovuto seguire il modello: gli Etoli - continua Polibio - non avrebbero potuto che approvarne ed ammirarne la megalopsychia, ed egli li avrebbe vinti non con le armi, ma con la rettitudine e nel diritto - come Filippo II aveva conquistato

\footnotetext{
16 Polibio XVIII 3, 4-7.

17 Sull'anchinoia in Polibio, la virtù del politico previdente, vd. M.-R. Guelfucci, Polybe et les mises en scène de la Tychè, op. cit., p. 443.
} 
le menti e i cuori degli Ateniesi. Sono queste le vittorie più vantaggiose - proseguiva Polibio -, perché i vinti si piegano per scelta, katà proairesin, e accettano di buon animo l'egemonia. Imboccata la via opposta rispetto ai modelli positivi di Filippo II, Alessandro e Antigono III, Filippo V col passare degli anni si procurò una fama contraria alla loro, e pari a quella degli etoli Scopa e Dorimaco, ai quali rimproverava di essersi macchiati di empietà a Dion e Dodona. Aveva scelto la strada del terrore, abbandonando la linea dei benefici, che a detta di Polibio gli avrebbero procurato sincera riconoscenza.

Al termine di una lunga riflessione, Polibio riprende il tema dell'influenza dei consiglieri su un re così giovane, già introdotto anche a proposito della rinuncia a punire gli Spartani nel 220: come lì aveva attribuito ad Arato la scelta più favorevole agli Spartani, così ora riversa su Demetrio di Faro la responsabilità della decisione di segno opposto - in base all' analogia con le risposte date dai due a Filippo sull'Itome. Nei tre casi che abbiamo esaminato, dunque, Arato e Demetrio di Faro finiscono per impersonare la contrapposizione fra la linea di un dominio tirannico, basato sulla violenza, o almeno sulla minaccia dell'impiego della forza, e quella volta invece a procurarsi con i benefici l'eunoia dei nemici vinti e dei popoli soggetti ${ }^{18}$. Che la preferenza di Polibio vada a questa seconda politica, è manifesto. Ė anche significativo che la sua condanna della condotta di Filippo V a Termo coincida con il programma politico cui diede voce l'etolo Agelao, nel corso dei negoziati che condussero alla pace di Naupatto, nel 217 a.C. (V 104, 5-6). Rappresentante di uno stato minore costretto a piegarsi di fronte a una superpotenza, Agelao delineò il modello di un'egemonia macedone sulla Grecia basata, ancora una volta, sulla pistis: rinunciando « a distruggere i Greci », il re avrebbe dovuto piuttosto conquistarsene l'eunoia, trasformandoli così in « sicuri collaboratori per i suoi disegni » - secondo il modello di Filippo II dopo Cheronea, esaltato più volte da Polibio. Le potenze straniere, impressionate dalla pistis dei Greci nei confronti del re, non avrebbero osato attaccarlo. La posizione di Agelao è la stessa che Polibio difenderà con enfasi oratoria commentando il comportamento di Filippo $\mathrm{V}$ a Termo. L'apparizione dei medesimi toni di condanna, più avanti, anche nel discorso dell' etolo Clenea $^{19}$, è un segno ulteriore della continua contaminazione fra storiografia e politica nelle Storie.

A raccomandare a Filippo V la linea dell'epieikeia e della pistis, celebrata da Polibio, erano stati Agelao di Naupatto e Arato, i leaders rispettivamente di un nemico vinto che

\footnotetext{
18 Forti dubbi sull'attribuzione ad Arato di consigli di moderazione a Filippo V a Termo esprime D. Golan, The res Graeciae in Polybius. Four Studies, Como, 1995, p. 31-32; sembra lecito estenderli anche alla questione del comportamento nei confronti della fazione filoetolica a Sparta nel 220.

19 Polibio IX 30, 2.
} 
rivendicava il trattamento più mite nel corso delle trattative di pace e di un alleato minore che voleva difendere i propri spazi di autonomia ${ }^{20}$. Fra i philoi del re, in questi stessi anni, oltre a Demetrio di Faro, anche altri, più immediatamente solleciti degli interessi della potenza dominante, andavano suggerendo la linea opposta: Apelle aveva raccomandato a Filippo la 'tessalizzazione' degli alleati, mostrandogli che, « se avesse prestato ascolto ad Arato, avrebbe trattato gli Achei nei termini dell' alleanza messa per iscritto, se invece avesse dato retta a lui $[. .$.$] , avrebbe trattato tutti i Peloponnesiaci come voleva ». Il loro obiettivo$ era costringere gli alleati a $\pi \circ เ \varepsilon \tilde{\imath} \tau$ condizione dei Tessali in rapporto alla Macedonia (IV 76, 2). La formula, ricorrente nelle Storie, merita di essere indagata; sembra esprimere una lettura realistica della sottomissione inerente ai rapporti di egemonia, con i suoi aspetti coercitivi: Demetrio di Faro, in V 101, 9, riportava al terrore derivante dall' esito della guerra la disponibilità degli Etoli ad obbedire (appunto, poieîn tò prostattomenon) a Filippo V; anche altrove, la sottomissione che si esprime nel poieîn tò prostattomenon è esplicitamente legata al terrore e alla costrizione (V $51,8)$. Non a caso, così, evitare di poieîn tò prostattomenon è l'obiettivo dichiarato della guerra degli Etoli contro Filippo V (XI 5, 1), di quella degli Achei contro Cleomene (IV 76, 7), o più tardi di quella dei Licii contro i Rodii (XXII 5, 10). Significativamente, come è stato rilevato, nelle relazioni internazionali le cancellerie ellenistiche preferivano al più esplicito prostattein il meno duro e più cortese parakaleîn, che consentiva di celare elegantemente l'imposizione sotto le vesti di una richiesta, facendo apparire il proprio dominio più accettabile, o meno umiliante la necessità di obbedire ${ }^{21}$; ma il ricorrere dell'espressione poieîn tò prostattomenon indica pure la diffusa consapevolezza degli aspetti più brutali dell'obbedienza e della sottomissione, che nel linguaggio ufficiale si preferiva tacere. Da ultimo, vale forse la pena rilevare l'analogia fra l'espressione poieîn tò prostattomenon e il latino imperium, che ha avuto maggior fortuna: in XXXV 3, 9 essa indica ad esempio l'obiettivo romano della sottomissione della Spagna. A riprova della sostanziale equivalenza

\footnotetext{
20 Per la necessità di intendere le posizioni di Polibio all'interno della tradizione del confronto fra la Lega achea e le potenze maggiori, a partire da Arato di Sicione, vd. A.M. Eckstein, Moral Vision in The Histories of Polybius, Berkeley-Los Angeles-London 1995, p. 197-198; 200, e già K. Ziegler, Polybios, RE, XXI, 2, 1952, 1440-1578, in particolare 1476 1477; D. Musti, Polibio negli studi dell'ultimo ventennio (1950-1970), ANRW, I, 2, Berlin-New York 1972, p. 1114-1181, in particolare 1114 .

21 Vd. A. Erskine, Expressions of Power in Polybius' Histories, in M. Mari e J. Thornton (a cura di), Parole in movimento, op. cit., p. 81-92, in particolare 91-92, con riferimento a $S I G^{3} 543,1.34$ (Filippo V esprime in termini di esortazione i propri ordini alla città di Larisa) e a Polibio XXIX 27, 6, dove significativamente Antioco IV attenua la durezza dell'ingiunzione

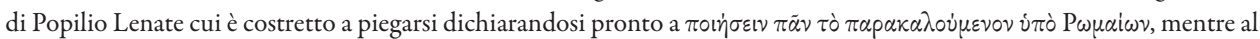

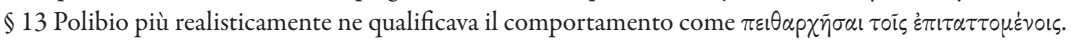


con il latino imperium, con il suo carico di obbedienza e sottomissione ${ }^{22}$, si può indicare infine un passo del discorso di Perseo ai suoi soldati alla vigilia della prima campagna di guerra, nel 171: nella versione liviana, in XLII 52, 16, l'alternativa a combattere per evitare l'asservimento ai superbi domini romani si esprime nella formula facere imperata - calco evidente del greco poiein tò prostattomenon. Anche qui, l'espressione indicava - o, piuttosto, denunciava - le ambizioni egemoniche altrui, un dominio dispotico al quale ci si sarebbe dovuti sottrarre anche a prezzo della guerra.

L'alternativa fra i due modi di esercizio del dominio fra i quali a più riprese si trovò a dover scegliere il giovane Filippo $V$ naturalmente non si era presentata nella storia greca solo con l'egemonia macedone, e Polibio non è il primo storico a rifletterla: basti pensare, in Tucidide, al dibattito sulla punizione di Mitilene, o al dialogo fra i Melii e gli Ateniesi ${ }^{23}$. Significativamente, in entrambi i casi - e altri se ne potrebbero aggiungere la linea dell'epieikeia viene invocata in difesa degli interessi di quanti si trovavano a dover fare i conti con la potenza imperiale; Cleone, per parte sua, annoverava l'epieikeia fra i tre vizi più dannosi a un impero ${ }^{24}$.

Polibio dunque fa sue le posizioni elaborate da una tradizione diplomatica che risaliva ben oltre l'età di Arato; e le sviluppa in una forma tipicamente retorica, attraverso il ricorso ai paradeigmata, secondo quanto previsto, e suggerito, già nella Rhetorica ad Alexandrum. Dapprima, Polibio riporta i paradeigmata addotti nell' ambiente di Filippo per indirizzarne o giustificarne il comportamento; quindi, vi ricorre in prima persona, per giudicarlo. Alla fine, imprigionato in questa rete di paradeigmata - fra i quali il più significativo appare quello di Filippo II dopo Cheronea, che ricorre ripetutamente nelle Storie ${ }^{25}$-, Filippo V assume anche lui valore paradigmatico. In VII 11, introducendo una digressione sul re macedone in occasione dell' inizio della sua degenerazione, Polibio affermava apertamente di considerare il caso di Filippo il paradeigma più evidente (enargestaton) per quanti, fra gli uomini politici, intendevano apprendere la lezione della storia. La condotta tenuta a Messene segna un momento di svolta nella carriera del re macedone: fino a quel momento, il giovane Filippo V era stato « il favorito di tutti i Greci, grazie al suo comportamento da benefattore »; da allora in poi, il re assunse invece « una condotta contraria a quella precedente »; e, inevitabilmente, cambiarono di segno

\footnotetext{
22 P. S. Derow, Polybius, Rome and the East, JRS, 69, 1979, p. 1-15, in particolare 4-6.

23 Qualche cenno in questa direzione già in Thornton, Polybius in Context, op. cit., p. 223-224; il tema, che qui può essere solo evocato, meriterebbe comunque di essere sviluppato altrove.

24 Tucidide III 40, 2.

25 Thornton, Polybius in Context, op. cit., p. 222.
} 
anche i giudizi degli altri su di lui. Dalla considerazione delle diverse fasi della carriera di Filippo, dunque, i lettori di Polibio avrebbero potuto trarre una lezione completa sulle forme più convenienti della gestione di un'egemonia: ai paradeigmata ek tôn homoíon, gli esempi da seguire, si affiancavano quelli ek ton enantion, i comportamenti da evitare.

La lezione potrebbe apparire conclusa, illustrata a sufficienza dagli esempi tratti dai primi anni del regno di Filippo V. La sua importanza agli occhi del maestro è tale tuttavia che Polibio non si stanca di ribadirla, replicando ripetutamente giudizi riconducibili alla linea di Arato e di Agelao: la centralità della figura di Filippo V nelle Storie sembra dipendere infatti anche dalla condivisione da parte di Polibio delle attese riposte nel re macedone dagli Achei, e andate amaramente deluse ${ }^{26}$. Rinunciando ad esaminare tutte le tappe della trasformazione di Filippo V nelle Storie, si può almeno rilevare come ogni qual volta si tocchi il tema cruciale dei rapporti con gli alleati Polibio avverta la necessità di intervenire in prima persona, mostrando una vena didascalica: a Creta, ad esempio, grazie all'applicazione della politica indicata da Arato (ancora una volta), Filippo « tenne in suo potere tutti i Cretesi e si conquistò il favore di tutti i Greci con la nobiltà della sua condotta $»^{27}$. Al contrario, in VIII 8 Polibio denuncia che quelli che a lui apparivano atti di asebeia e paranomía venivano esaltati da quanti avevano scritto intorno a Filippo V; a simili posizioni, Polibio negava ogni legittimità: le riportava al favore o al terrore nei confronti del re e le escludeva dal campo della storiografia, degradandole al rango dell'encomio ${ }^{28}$. L'immediata apertura di una digressione sulla rappresentazione dei re, di cui fa le spese Teopompo, appare un tentativo di spostare sul piano del metodo storico contrasti che erano invece essenzialmente politici: lo dimostra l'apparizione degli stessi toni di condanna del comportamento di Filippo $V$ a Termo e a Messene nel discorso dell'etolo Clenea ${ }^{29}$, e la sua giustificazione nella replica dell' acarnano Licisco, filomacedone ${ }^{30}$.

I commenti di Polibio accompagnano anche le fasi successive della carriera di Filippo: a proposito del suo comportamento nei confronti della città bitinica di

\footnotetext{
26 Cf. già M.-R. Guelfucci, Polybe et les mises en scène de la Tychè, op. cit., p. 461.

27 Polibio VII 14, 4.

28 Per la distinzione fra encomio e storiografia in Polibio vd. già X 21, 5-8, a proposito di Filopemene, e cf. da ultimo S.T. Farrington, Action and Reason: Polybius and the Gap between Encomium and History, CPh, 106, 2011, p. 324-342.

29 Polibio IX 30, 1-2, in cui si rilevi la concentrazione in poche righe di termini quali paranomía, asebeia, hybreis, omotes, athesía e paraspondesis - un vero campionario dell'orrore.

30 Polibio IX 35, 6, con il rovesciamento delle accuse di hybris e paranomía sugli Etoli, per il loro comportamento a Dion e Dodona, che giustificherebbe la reazione di Filippo V.
} 
Cio, nel 202, Polibio contesta ancora una volta i motivi dell'autorappresentazione di Filippo, e ne mette in ridicolo la convinzione di aver compiuto « un'impresa nobile e rispettabile $»^{31}$. L'elemento centrale nel contrasto fra la linea seguita dal re e le critiche di Polibio è sempre quello del modo più idoneo ad ampliare e conservare l'egemonia: la soddisfazione di Filippo per « aver terrorizzato tutti i suoi avversari $»^{32}$ rimanda alla concezione del terrore come strumento di estensione e consolidamento del dominio - quella già incontrata nella distruzione di Tebe da parte di Alessandro, additata a modello a Filippo fin dalla sua prima campagna nel Peloponneso. Per parte sua, Polibio contesta radicalmente l'efficacia di questa via, e condanna il comportamento di Filippo a Cio non solo sul piano morale ${ }^{33}$, ma anche su quello dei risultati pratici: con la campagna contro Lisimachia, Calcedone e Cio il re s'era procurato l'ostilità dei Rodii e l'odio degli Etoli, e in definitiva aveva dimostrato a tutti la propria « slealtà e inaffidabilità » (athesía e abebaiotes). Un re che nutriva grandi ambizioni avrebbe dovuto evitare di dare tali precoci prove di omotes e asebeia, alienandosi fin dall' inizio i popoli che avrebbe dovuto trarre dalla sua trattandoli da amici ed alleati ${ }^{34}$, ed escludendo così ogni possibilità di costruire un rapporto basato sulla pistis.

Per Polibio, come già, comprensibilmente, per Arato o Agelao di Naupatto, a garantire la stabilità del dominio, attraverso la riconoscenza che ne derivava, l'eunoia, dovevano essere i benefici nei confronti degli alleati minori, e persino dei nemici vinti, da trasformare per questa via in fedeli alleati. In questa prospettiva Polibio presenta anche l'episodio dell'etolo Nicandro, che nell'estate del 191, di ritorno dall'Asia, dove aveva sollecitato il soccorso di Antioco III, rifugiatosi nel suo regno dopo la sconfitta delle Termopile, cadde prigioniero dei Macedoni e venne portato di fronte al re. Contro ogni aspettativa, Filippo V lo trattò con generosità, gli trasmise un messaggio politico di carattere panellenico in funzione antiromana, e lo invitò « a ricordarsi del beneficio a lui reso $\gg^{35}$; da allora in avanti, quasi a dimostrare l'efficacia della linea della mitezza, che produce l'eunoia verso la potenza egemone, Nicandro si mantenne sempre « fedele alla casa reale dei Macedoni »; al tempo della guerra contro Perseo, i Romani, non potendosene fidare, lo deportarono a Roma, dove morì - probabilmente, dopo aver

\footnotetext{
31 Polibio XV 22, 1.

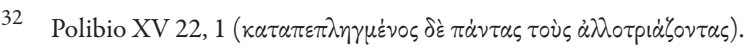

33 Polibio XV 22, 2.

34 Polibio XV 24, 4-6.

35 Polibio XX 11, 8.
} 
avuto il tempo di narrare la sua avventura a Polibio, che si affrettò a trarne la lezione per i lettori.

Particolarmente istruttiva, agli occhi di Polibio, doveva risultare anche la storia del breve dominio di Filippo V sull'Atamania, all'epoca della sua alleanza con i Romani, nella guerra contro Antioco III e gli Etoli; le vicende della trasmissione del testo delle Storie fanno sì che la si possa ricostruire solo attraverso Livio. All'inizio delle operazioni del 191 a.C., il console Manio Acilio affidò a Filippo i prigionieri atamani delle guarnigioni che si erano arrese. Filippo li trattò con indulgenza, per conciliarsi per mezzo loro il popolo degli Atamani; condotto l'esercito nella regione, liberò i prigionieri, rimandandoli nelle loro città, dove essi celebrarono la clementia e la munificentia mostrate dal re macedone. Aminandro, inquieto, temendo di essere consegnato a Filippo e ai Romani, abbandonò il regno, rifugiandosi ad Ambracia; l'Atamania, così, passò ai Macedoni (Livio XXXVI 14, 3-9). Conquistata da Filippo grazie al favore procuratosi con la generosità nei confronti dei prigionieri, la regione fu persa, nel 189, a causa del superbum atque immodicum imperium esercitatovi dai suoi praefecti, che suscitò nostalgia di Aminandro; la popolazione avrebbe aderito compatta alla rivolta organizzata dai fautori del re esule; i presidii macedoni furono scacciati, e Aminandro, con l'aiuto degli Etoli, poté recuperare il regno (Livio XXXVIII 1). Nel breve volgere di un paio d'anni, così, Filippo aveva fornito ancora una volta una duplice lezione: come acquistare il potere con la clementia e perderlo a causa della superbia.

Infine, nel 185 a.C., a Tempe, di fronte a un'ostile commissione senatoria incaricata di dare ascolto alle lamentele contro Filippo dei suoi vicini della Grecia centro-settentrionale, alcuni degli ambasciatori tessali avrebbero rivolto al re macedone l'invito ut deposita domini acerbitate adsuesceret socium atque amicum sese praestare, et imitaretur populum Romanum, qui caritate quam metu adiungere sibi socios mallet ${ }^{36}$. In un passo di chiara derivazione polibiana, emerge ancora una volta, con piena evidenza, la portata retorico-diplomatica dell'esempio di quanti si procuravano con i benefici la fedeltà degli alleati, e la contrapposizione di questa linea politica a quella opposta, del dominio dispotico esercitato attraverso il terrore. Ma ormai, gli esempi da seguire additati a Filippo $\mathrm{V}$ non derivano più dalla storia interna della dinastia macedone; è ai temi dell' autorappresentazione romana che si ispirano gli abili rappresentanti dei Tessali. Ancora una volta però, come già nei casi di Arato e Agelao di Naupatto, a suggerire la linea che qui potremmo definire della caritas sono 'alleati' recalcitranti, desiderosi

36 Livio XXXIX 25, 14-15. 
almeno di un trattamento più mite, che pur nell'ambito di un formale riconoscimento dell'egemonia garantisse loro ampi spazi di autonomia.

Il senso dell' insistenza di Polibio su questi motivi non si esaurisce però nell'ostinata riproposizione delle aspettative achee sui rapporti con i re macedoni; Filippo non è infatti l'unico esempio che Polibio propone ai suoi lettori.

\section{- II -}

Dalla Macedonia, dunque, spostiamoci a Cartagine. L'imperialismo punico è analizzato a più riprese nelle Storie, già a partire dalla prokataskeué, nel primo libro. Polibio sembra voler approfittare della guerra contro i mercenari e le popolazioni libiche ribelli per esporre le sue riflessioni sui rischi inerenti a un esercizio del dominio improntato a eccessiva durezza. Lo spazio fatto a questa vicenda e la frequenza con cui Polibio interrompe la narrazione degli eventi per trarne le opportune lezioni suggeriscono l'urgenza di esprimere fin dall' inizio dell'opera alcune delle idee chiave delle Storie ${ }^{37}$; e tra queste, innanzi tutto, proprio il messaggio della pericolosità, per una potenza egemone, della linea della pikría. Secondo i precetti della Rhetorica ad Alexandrum, Polibio si sofferma sulla vicenda per fornire precocemente ai suoi lettori un esempio ek tôn enantion.

La rivolta delle popolazioni libiche aveva minacciato l'esistenza stessa di Cartagine; per Polibio, la si sarebbe potuta evitare, rinunciando a quegli eccessi nello sfruttamento che avevano esacerbato gli animi delle genti soggette. Sarebbe bastata un po' di previdenza; sarebbe bastato scegliere praotes e philanthropia, in luogo della pikría:

Proprio loro, soprattutto, erano stati causa per se stessi di tali e tanto grandi mali. Durante la guerra precedente, infatti, pensando di avere ragionevoli pretesti per farlo, avevano esercitato duramente il loro potere sulle genti della Libia, sottraendo la metà di tutti gli altri raccolti $\mathrm{e}$ imponendo alle città tributi doppi rispetto a prima, e non concedendo assolutamente ai poveri alcuna indulgenza o esenzione da nessuno dei pagamenti, ammirando e onorando sempre, tra i governanti, non quelli che trattavano il popolo con dolcezza e umanità, ma quelli che procuravano loro la massima quantità di risorse e di approvvigionamenti, e che trattavano con la massima asprezza gli abitanti della regione (tra quelli che agivano così c'era Annone). Perciò gli uomini non ebbero bisogno nemmeno di un incoraggiamento alla ribellione, ma solo di

\footnotetext{
37 Già K.-E. Petzold, Studien zur Methode des Polybios und zu ibrer historischen Auswertung, München, 1969, p. 22-23 aveva osservato come la rivolta dei mercenari fosse stata inserita nella prokataskeuè, oltre che perché consentiva di cogliere le cause della guerra annibalica (il motivo principale dichiarato da Polibio in I 65, 5-9), in ragione « der Beantwortung anderer den Politiker interessierender Fragen ». Che Polibio abbia impiegato la prokataskeue per esprimere fin dall'inizio le idee che più gli stavano a cuore è anche l'opinione di H. Beck, Polybius' Roman prokataskeuē, in B. Gibson, Th. Harrison (ed. by), Polybius and his World, op. cit., n. 7, p. 125-142, in particolare 141-142.
} 
un annuncio; da parte loro le donne, che in precedenza permettevano che i loro mariti e i loro padri fossero condotti in prigione per le tasse, e che ora giuravano insieme, città per città, di non nascondere nulla di quanto possedevano, privandosi dei loro ornamenti contribuivano senza esitazioni alla paga dei soldati. E assicurarono tali entrate a Mato e Spendio che essi non solo pagarono ai mercenari quanto ancora era loro dovuto del soldo secondo le promesse che avevano fatto loro per convincerli alla ribellione, ma ebbero anche abbondanza di mezzi per il periodo successivo. Così, bisogna sempre che chi prende decisioni giuste non guardi mai solo al presente, ma anche e soprattutto al futuro $(\mathrm{I} 71,8-72,8)$.

Alla politica miope dei Cartaginesi, con i suoi effetti rovinosi, Polibio avvertì l'esigenza didattico-retorica di contrapporre, nella stessa Cartagine, un diverso modello di esercizio del dominio, più prudente e produttivo. Anche la vicenda cartaginese poteva fornire non solo esempi ek tôn enantion, ma anche ek tôn homoíon. Fra i prigionieri di guerra, Amilcare Barca arma ed arruola nel suo esercito i volontari, e perdona gli altri, lasciandoli liberi di andare dove desiderassero (I 78, 13-14). Allarmati da questa politica, i capi degli insorti temono che la generosità di Amilcare possa minare la compattezza del fronte dei ribelli, e spingono i loro uomini a macchiarsi di delitti tanto gravi da escludere ogni possibilità di riconciliazione con i Cartaginesi; è a partire da questo momento che il conflitto assume il carattere di un aspondos polemos ${ }^{38}$.

Come ad Atene, con Aristide e Pericle da una parte, Cleone e Carete dall'altra, a Sparta, con Cleombroto e Agesilao, e nella corte di Filippo V, con Arato e Demetrio di Faro, dunque, anche a Cartagine Polibio contrappone una politica dura, che genera l'odio e la rivolta dei popoli soggetti, a una linea mite, umana, generosa, che produce invece l'effetto opposto, ed è l'unica a poter garantire la stabilità del dominio.

Di questo, che può considerarsi il cardine del suo messaggio politico, Polibio troverà più avanti una significativa conferma nell'analisi di un altro momento di crisi del dominio cartaginese. In Spagna, nel 211, la sconfitta degli eserciti di Publio e Gneo Cornelio Scipioni aveva ridotto i Romani in una condizione disperata - almeno nella valutazione di Polibio ${ }^{39}$; ma al futuro Africano, che appena venticinquenne aveva ottenuto il comando nella penisola iberica, Polibio attribuisce ben fondate speranze di successo: i Cartaginesi, infatti, « trattando gli alleati con tracotanza, se li erano alienati e inimicati tutti ». Per vendicarsi della loro arroganza, le popolazioni iberiche erano pronte a passare

\footnotetext{
Polibio I 79, 8-81, 11.

39 Che potrebbe però essere un po' esagerata: A. Erskine, Spanish Lessons: Polybius and the Maintenance of Imperial Power, in J. Santos Yanguas and E. Torregaray Pagola (eds.), Polybius y la península ibérica, Revisiones de Historia Antigua, IV, Vitoria-Gasteiz, 2003, p. 229-243, in particolare 229-230.
} 
dalla parte dei Romani, « non tanto perché ben disposti verso di loro, ma piuttosto nel desiderio di vendicarsi dell' arroganza dei Cartaginesi nei loro confronti » (X 6, 3-4).

La parabola del dominio punico in Iberia, dalle vittorie del 211 al crollo del sistema di alleanze con i popoli indigeni dopo la presa di Cartagine Nuova da parte di Scipione, fornisce a Polibio l'occasione di riproporre quella teoria sui rapporti fra potenza egemone e popoli soggetti che aveva sentito l'esigenza di esporre fin dal primo libro:

Dopo aver vinto le truppe romane e aver ucciso entrambi i generali - Publio e Gneo -, infatti, ritenendo di avere ormai un controllo incontrastato dell'Iberia, cominciarono a trattare con insolenza le genti della regione. Pertanto nei loro sudditi ebbero non alleati ed amici, ma nemici. Ed è naturale che patissero questa condizione: appunto per aver creduto che gli imperi si conquistano in un modo e si conservano in un altro modo, non impararono che difendono meglio la supremazia coloro che meglio si mantengono fedeli agli stessi principî con i quali hanno originariamente conquistato i loro dominî. Eppure è evidente, ed è stato già osservato in molti casi, che gli uomini guadagnano le posizioni di potere beneficando il prossimo e offrendogli buone speranze, ma quando, realizzati i loro desideri, fanno del male ai loro sudditi e li governano in forma dispotica, le inclinazioni dei sudditi mutano, come è naturale, di pari passo con i mutamenti in chi comanda. Ciò avvenne anche allora ai Cartaginesi (X 36, 3-7).

Convinti di detenere ormai il dominio incontrastato dell'Iberia, dunque, i Cartaginesi presero a trattare con insolenza le popolazioni locali; e il loro dispotismo trasformò i popoli soggetti da alleati ed amici in nemici. La lezione che Polibio trae dalla vicenda è che il dominio incontrastato, aderitos, porta con sé i rischi dell'hyperephanía, che inevitabilmente genera i risentimenti dei popoli soggetti, e può produrne la rivolta ${ }^{40}$.

A presentare queste considerazioni fu un politico acheo, costretto ad assumere le vesti dello storico dalla sua deportazione a Roma, al termine della terza guerra di Macedonia. Fin dal primo capitolo del primo libro delle Storie, Polibio rilevò il carattere paradoxon della rapida conquista romana di quasi tutto il mondo abitato ${ }^{41}$. Con il 168 , venuto meno il regno di Macedonia, faceva iniziare la aderitos exousía romana (XXXI 25, 6); in termini più alla moda, si è parlato di passaggio ad una situazione gerarchica ed unipolare ${ }^{42}$. Aderitos,

\footnotetext{
40 Sul brano cf. J. de Romilly, La douceur dans la pensée grecque, Paris, 1979, p. 241; J.-L. Ferrary, Philhellénisme et impérialisme. Aspects idéologiques de la conquête romaine du monde hellénistique, de la seconde guerre de Macédoine à la guerre contre Mithridate, École Française de Rome 1988, p. 350; A. Erskine, The Hellenistic Stoa: political thought and action, London 1990, p. 185; A.M. Eckstein, Moral Vision, op. cit., p. 227-229, e soprattutto la fine analisi di A. Erskine, Spanish Lessons, op. cit., p. 231-232.

41 Polibio I 1, 4-5; cf. XXXIX 8, 7.

42 A. Eckstein, Rome Enters the Greek East. From Anarchy to Hierarchy in the Hellenistic Mediterranean, 230-170 BC, Malden, MA, 2008.
} 
'incontrastata', è comunque lo stesso aggettivo impiegato per definire il dominio punico nella penisola iberica dopo la rotta degli Scipioni, nel $211^{43}$; una situazione che aveva portato alla hybris e alla hyperephanía, con la conseguente rivolta dei popoli soggetti e la perdita dell'egemonia. In VI 57, 5 poi, nel libro teorico, Polibio aveva considerato i rischi di degenerazione che minacciavano una politeia che avesse conseguito byperochèn kai dynasteian aderiton: non a caso, il passo è stato spesso inteso come un riferimento a Roma ${ }^{44}$.

$\mathrm{Su}$ questi temi Polibio non riflette con lo sguardo distaccato dell'osservatore neutrale, del teorico delle relazioni internazionali, ma a partire dalla sua condizione di Greco soggetto all'egemonia romana, e nel solco di tutta una tradizione politicodiplomatica sviluppatasi nel confronto con le potenze egemoni. È la tradizione di Arato, nella gestione dei rapporti con Filippo V, è la tradizione della disperata difesa diplomatica della lettera dei trattati di fronte alle potenze egemoni: una linea per la quale Polibio esprime il proprio apprezzamento lungo tutto l'arco cronologico coperto dalle Storie, che si tratti di Ierone II di Siracusa ${ }^{45}$, di Arato, di Filopemene ${ }^{46}$ o di Cefalo d'Epiro ${ }^{47}$. Da Arato, Filopemene e Licorta, Polibio eredita l'aspirazione a un'egemonia benevola fondata sulla pistis, che rispetti scrupolosamente i trattati, nonostante lo squilibrio nei rapporti di forza. $\mathrm{E}$ in questa tradizione diplomatica che si deve intendere la teoria polibiana dell'impero - o piuttosto, le riflessioni sul tema dell'egemonia e dell'impero che Polibio sottopone ai propri lettori. Essa non nasce in una biblioteca, rifugio di uno studioso da tavolino, ma nell'agone diplomatico, nel tentativo di difendere i propri spazi di autonomia pur nella necessaria sottomissione alle aspirazioni egemoniche di una grande potenza. Quando Polibio riflette amaramente sull'esperienza storica di Filippo V, o esalta la magnanimità di Filippo II, e ancor più quando analizza i momenti di crisi del dominio punico in Africa e poi in Iberia, lo scopo principale che si propone è inviare un messaggio ai suoi lettori, e in particolare alla classe dirigente romana. D'altra parte, che nel discorso politico greco contemporaneo la riflessione sulle esperienze egemoniche precedenti, e in particolare sull' impero ateniese e l'egemonia spartana, venisse impiegata

43 Cf. ora G. Zecchini, Ad́ńpıros in Polibio, in M. Mari e J. Thornton (a cura di), Parole in movimento, op. cit., p. 93-98.

44 Cf. per esempio J. Thornton, La costituzione mista in Polibio, in D. Felice (a cura di), Governo misto. Ricostruzione di un'idea, Napoli, 2011, p. 67-118, in particolare 87 n. 49; 117-118.

45 Vd. Polibio I 83, 2-4, con A.M. Eckstein, Polybus, Syracuse, and the Politics of Accomodation, GRBS, 26, 1985, p. $265-282$.

46 Cf. per esempio in Polibio XXII 10, 8 il succo dei discorsi di Filopemene, Licorta e Arcone al legato Quinto Cecilio Metello nel 185 (« ... non era possibile cambiare nulla, nella situazione presente, senza venir meno agli obblighi verso gli uomini e al sacro rispetto degli dèi »).

47 Vd. Polibio XXVII 15, 10-12, e più in generale, sulla posizione della optima pars della classe dirigente greca alla vigilia della guerra contro Perseo, cf. Livio XLII 30, 5-7. 
in rapporto al dominio romano, lo testimoniano i quattro logoi dell'opinione pubblica greca sulla terza guerra punica riportati nel celebre capitolo XXXVI $9^{48}$.

Arnaldo Momigliano scrisse che Polibio « aveva passato la maggior parte del suo tempo spiegando ai Greci e ai Romani le ragioni per cui questi ultimi erano destinati alla vittoria »; solo il suo successore Posidonio avrebbe tentato di impartire sistematicamente ai Romani una lezione di moderazione, non esitando a «parlare apertamente per ricordare ai governanti romani i loro errori e crimini $\gg{ }^{49}$. In realtà, il tentativo di indicare al senato la linea da seguire è già nel DNA politico di Polibio, in cui si era sedimentata la tradizione del confronto delle poleis minori con le potenze egemoni. La teoria secondo cui la linea della mitezza sarebbe la più utile dal punto di vista della stessa potenza imperiale, di cui parallelamente si esaltano le virtù morali, è un'arma diplomatica, prima e più che uno strumento di analisi politologica. Se Polibio vi insiste fin dal primo libro, se è a questi principi che impronta il suo giudizio su Filippo V, personaggio centrale nelle Storie, se vi ritorna poi in modo quasi ossessivo ogni qual volta se ne presenti l'occasione, è per tentare di indirizzare i comportamenti concreti della classe dirigente romana. La riflessione sugli imperi, per un politico educato nelle scuole di retorica, non è che la moltiplicazione dei paradeigmata, tanto ek tôn enantion quanto ek tôn homoion, accumulati per aumentarne l'effetto persuasivo; le Storie assumono così il carattere di un discorso diplomatico ${ }^{50}$. Conseguita l'aderitos exousía nel Mediterraneo, il senato, come i Cartaginesi in Spagna, poteva cedere alla tentazione di trattare i popoli soggetti con hyperephania e hybris; richiamando in ogni possibile occasione gli effetti rovinosi della hybris nei confronti degli alleati e il successo della linea di condotta opposta, quella dell'epieikeia, l'opera storiografica dell'acheo Polibio si sforza di mettere in guardia la classe dirigente romana da questi rischi.

Anche la costruzione della figura di Scipione Africano mira a presentare ai lettori romani un esempio da seguire: la sua generosità nei confronti degli ostaggi iberici a Cartagine Nuova presa nel 209, l'innocenza per cui stenta persino a capire quali oltraggi avessero subito « le figlie di Andobale e di parecchi altri dinasti », tenute in ostaggio dai Cartaginesi, la promessa di prendersene cura « come avrebbe fatto con le proprie sorelle e le proprie figlie », e infine la restituzione al padre della fanciulla di cui i suoi soldati

\footnotetext{
48 Vd. in particolare Polibio XXXVI 9, 5.

49 A. Momigliano, Saggezza straniera. L'Ellenismo e le altre culture, Torino, 1980, p. 38-39 (ed. orig. Alien Wisdom. The Limits of Hellenization, Cambridge, 1975, p. 36).

50 Cf. Thornton, Polybius in Context, op. cit., p. 213-214 e passim.
} 
avrebbero voluto fargli dono contribuiscono a renderlo caro alle popolazioni iberiche $\mathrm{e}^{51}$. La virtù di Scipione non tarda a produrre risultati positivi:

Andobale e Mandonio, che erano allora i dinasti più importanti dell' Iberia e avevano fama di essere i più sinceri amici dei Cartaginesi, nutrivano invece un segreto rancore e da tempo erano in attesa di un'occasione: da quando cioè Asdrubale, con il pretesto di non fidarsi di loro, aveva richiesto loro una notevole somma di denaro e le mogli e le figlie in ostaggio, come abbiamo mostrato in precedenza; in quel momento, ritenendo di avere un'occasione favorevole, presero con loro le proprie truppe e uscirono di notte dal campo cartaginese per ritirarsi in certi luoghi forti, in grado di garantire loro la sicurezza. Dopo questo fatto, Asdrubale fu abbandonato anche dalla maggior parte degli altri Iberi, che si sentivano da tempo oppressi dall' arroganza dei Cartaginesi e sfruttarono allora la prima occasione per far uscire le loro inclinazioni allo scoperto (X 35, 6-8).

Passando dalla parte del generoso Scipione, le popolazioni iberiche fanno emergere, e traducono in un gesto concreto il segreto rancore nutrito da tempo nei confronti dei Cartaginesi $^{52}$. Nel X libro delle Storie, così, Polibio non si limita ad affiancare, con Scipione, un esempio ek tôn homoín agli esempi ek tôn enantion forniti dai Cartaginesi, ma può persino presentare ai suoi lettori i risultati di una sorta di esperimento - che confermano non direi tanto la sua 'teoria', quanto la necessità di adottare la linea da lui suggerita. Nel laboratorio storico dell'Iberia, ad assicurarsi la fedeltà delle popolazioni indigene non sono i Cartaginesi, con la loro bybris, ma le virtù di segno opposto di cui diede prova Scipione.

In conclusione, Polibio va inteso all'interno della tradizione diplomatica a cui apparteneva. Praotes, epieikeia, philanthropia nei confronti degli alleati erano virtù rivendicate nell'autorappresentazione dei Romani ${ }^{53}$; Polibio li esorta ad applicarle davvero. In questo spazio angusto, che era andato vieppiù restringendosi col passaggio dall'equilibrio di potenze all'aderitos exousía romana, che aveva segnato un drammatico indebolimento della forza contrattuale degli stati minori, si erano dovuti muovere il suo eroe Filopemene e suo padre Licorta, nella gestione dei rapporti con Roma; in questo

\footnotetext{
51 Polibio X 18, 3-19, 7.

52 A illustrare simili dinamiche può essere utile la riflessione di James C. Scott, Domination and the Arts of Resistance. Hidden Transcripts, New Haven-London, 1990; cf. già J. Thornton, Terrore, terrorismo e imperialismo. Violenza e intimidazione nell'età della conquista romana, in G. Urso (a cura di), Terror et pavor. Violenza, intimidazione, clandestinità nel mondo antico. Atti del Convegno internazionale Cividale del Friuli, 22-24 settembre 2005, Pisa, 2006, p. 157-196.

Cf. per esempio Polibio XV 17, 4 per la decisione di Scipione Africano di trattare i Cartaginesi, nonostante le violazioni del trattato imputate loro, praos e megalopsychos; inoltre, almeno per praotes e philanthropia, vd. anche Polibio XXVIII 3, 3, o le parole attribuite a Flaminino in Polibio XVIII 37, 7 sulla necessità di mostrarsi nella vittoria $\mu \varepsilon \tau$ piovs

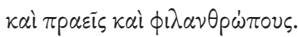


stesso spazio si era svolta la sua azione politica al tempo della terza guerra di Macedonia; ed è ragionevole ritenere che vi si debbano collocare anche le Storie. Il contesto in cui va inserita la redazione delle Storie ${ }^{54}$ è l'ambiente politico in cui Polibio era stato allevato e aveva fatto le sue prime prove, nei tempi difficilissimi della terza guerra di Macedonia, e il problema perdurante delle relazioni con l'egemonia romana, e della salvaguardia degli spazi di autonomia del mondo greco. Così, spiegare l'attività e le finalità perseguite da Polibio attraverso un breve brano della Rhetorica ad Alexandrum risulta in definitiva più legittimo che attribuirgli una distaccata riflessione politologica sulle modalità dell' acquisto e del mantenimento di un impero ${ }^{55}$. Anche chi esitasse ad attribuire a Polibio il consapevole perseguimento di finalità politico-diplomatiche, secondo quanto si è tentato di dimostrare, o almeno di suggerire qui, non potrà negare però un legame fra la 'riflessione' dello storico sugli imperi e l'azione politica degli achei Arato, Filopemene e Licorta nei confronti dell' egemonia macedone prima, di quella romana poi; derubricarlo a livello di riproposizione automatica, non del tutto intenzionale, di schemi interiorizzati al tempo della formazione dello storico implicherebbe una grave sottovalutazione delle capacità politiche dimostrate da Polibio in tutto il corso della sua vita.

\section{- III -}

Per la generazione di Polibio, per lo storico e i suoi lettori, la superpotenza con cui ci si doveva confrontare era ormai unicamente l'imperium Romanum; così, la questione del giudizio di Polibio su Roma e il suo dominio è divenuta un tema classico della ricerca sul mondo ellenistico-romano. L'intensa frequentazione dell'argomento non ha portato però a risultati condivisi, tanto che ancora si stenta a sottrarsi alla sterile contrapposizione tra le immagini inconciliabili di un Polibio convertitosi a Roma e di un segreto ma tenace oppositore dell'egemonia romana ${ }^{56}$.

\footnotetext{
54 Vd. già D. Musti, Polibio e l'imperialismo romano, Napoli, 1978, p. 144.

55 Solo su questo punto mi distacco dall'analisi finissima di A. Erskine, Spanish Lessons, op. cit., che ha ispirato queste pagine più di quanto non risulti dal numero limitato delle citazioni. Sulla linea di Erskine, almeno nell' attribuire a Polibio il tentativo di spiegare le ragioni della sopravvivenza o del declino degli imperi, vd. anche J.-L. Ferrary, Le jugement de Polybe sur la domination romaine: état de la question, in J. Santos Yanguas and E. Torregaray Pagola (eds.), Polybius y la península ibérica, op. cit., p. 15-32, in particolare 19 n. 12 (e già Idem, Philhellénisme et impérialisme, op. cit., p. 350); a testimonianza che le distanze fra le diverse posizioni sono meno marcate di quanto potrebbe apparire, però, Ferrary invita anche ad « analyser le message que Polybe veut faire passer à travers ses Histoires », e coglie felicemente l'appello ai Romani a non cadere nella tentazione della philarchía per garantire la durata del loro impero (p. 30).

56 Per il dibattito sul tema a partire dagli anni Settanta del secolo scorso vd. J. Thornton, Polibio e Roma. Tendenze negli studi degli ultimi anni, Studi Romani, 52, 2004, p. 108-139; 508-525. Fra gli studi più recenti, vd. J.-L. Ferrary, Le jugement de Polybe sur la domination romaine, op. cit.; B. Virgilio, Polibio, il mondo ellenistico e Roma, Athenaeum, 95 , 2007, p. 49-73.
} 
Tuttavia, se si vuole comprendere il senso delle Storie nel contesto in cui furono concepite e diffuse, il fine da perseguire non è tanto decidere se Polibio si debba collocare nello scaffale dei filoromani o in quello opposto, ma tentare di cogliere gli obiettivi politici che Polibio si proponeva, nel dialogo diplomatico fra il mondo greco e Roma, intorno alla metà del II secolo a.C.

Così, rinunciando al tentativo di stabilire in astratto il segno - positivo o negativo - del giudizio di Polibio su Roma, si è tentato qui di individuare piuttosto il messaggio che Polibio intendeva trasmettere. La necessaria considerazione della facies retorica della sua formazione, e della sua personalità, consente di intendere i frequenti giudizi su esperienze egemoniche confrontabili con quella romana che affiorano nelle Storie come paradeigmata, strumenti di un discorso volto alla persuasione. Da un capo all'altro dell'opera, tutti gli sforzi di Polibio convergono nel tentativo di indurre la potenza egemone a un esercizio moderato del proprio potere. Aver stabilito questo fatto è, credo, più significativo di apporre un'etichetta. 